ADVERSE DRUG REACTION

\title{
Metformin induced acute pancreatitis precipitated by renal failure
}

\section{S Mallick}

Postgrad Med J 2004;80:239-240. doi: 10.1136/pgmj.2003.011957

Metformin is a biguanide commonly used in type 2 diabetes and considered to be a safe drug with minimal side effects. Approximately $2 \%$ of cases of acute pancreatitis may be caused by drugs, but it is not a known complication of mefformin therapy. To date only one case of pancreatitis has been reported in association with metformin, but that was secondary to metformin poisoning (overdose). This is the first reported case of pancreatitis caused by a therapeutic dose of metformin (although in this case renal failure precipitated the metformin toxicity). Severe lactic acidosis is a rare but life threatening complication of metformin, which occurs particularly in patients with renal failure.

A 61 year old woman presented to the emergency department with a history of vomiting for four days and abdominal pain for one day. Her medical history included type 2 diabetes, ischaemic heart disease, and previous myocardial infarction, and regular medications included aspirin, atenolol, clopidogrel, amlodipine, lisinopril, nitrate, metformin, nateglinide, and ibuprofen. Owing to vomiting, she had stopped taking most of her medications except the antidiabetic drugs. On clinical examination she looked dry and afebrile, and her initial blood pressure was $110 / 50 \mathrm{~mm} \mathrm{Hg}$, her pulse rate was 50 beats/min and regular, and she had an oxygen saturation of $98 \%$ on 2 l of oxygen. Abdominal examination revealed epigastric tenderness without guarding or rigidity. Blood tests showed haemoglobin of $129 \mathrm{~g} / \mathrm{l}$, a white cell count of $11.8 \times 10^{9} / \mathrm{l}$, urea of $28.8 \mathrm{mmol} /$ l, creatinine of $516 \mathrm{umol} / \mathrm{l}$, sodium of $145 \mathrm{mmol} / \mathrm{l}$, potassium of $6.7 \mathrm{mmol} / \mathrm{l}$, chloride of $95 \mathrm{mmol} / \mathrm{l}$, glucose of $9 \mathrm{mmol} / \mathrm{l}$, and lactate of $25 \mathrm{mmol} / \mathrm{l}$. Arterial blood gases showed a $\mathrm{pH}$ of 7.0 and an anion gap of $43 \mathrm{mmol} / \mathrm{l}$. A liver function test was grossly normal. Amylase was raised at $250 \mathrm{U} / \mathrm{l}$, and this subsequently increased to $769 \mathrm{U} / \mathrm{l}$. Computed tomography of the abdomen was suggestive of pancreatitis. Her renal function had been checked one month previously and had been normal, and her glycated haemoglobin was 5.9\%.

She deteriorated further in the emergency department, with blood pressure dropping to $65 / 50 \mathrm{~mm} \mathrm{Hg}$ and a urine output of less than $5 \mathrm{ml} /$ hour. Bicarbonate infusion did not improve her acidosis. She was transferred to the intensive care unit and treated with intravenous fluids, ionotrope, insulin, and haemodialysis with bicarbonate buffered replacement fluid. All medications were discontinued. Lactic acidosis resolved following haemodialysis, and her amylase returned to a normal level.

\section{DISCUSSION}

This woman was diagnosed with drug (angiotensin converting enzyme inhibitor, non-steroidal anti-inflammatory drug) induced renal failure, which reduced excretion of metformin, causing toxicity leading to severe lactic acidosis and pancreatitis.

Severe lactic acidosis is a rare but life threatening complication of metformin therapy, occurring particularly in patients with relative contraindications to the drug (renal dysfunction, alcohol abuse, or liver disease). The mortality rate of metformin associated lactic acidosis is about 50\%, although available data show that neither arterial lactate level nor plasma metformin concentration are of prognostic significance.

The common causes of pancreatitis, such as gall stones, alcohol abuse, hypercalcaemia, hypertriglyceridaemia, and trauma, were excluded in this case. A number of drugs (thiazides, azathioprine, oestrogens, sulfonamides, furosemide, etc) can cause pancreatitis and are thought to be responsible for approximately $2 \%$ of pancreatitis in adults. ${ }^{2}$ None of these drugs was consumed by this patient except for an angiotensin converting enzyme inhibitor, which is occasionally associated with pancreatitis, ${ }^{3}$ which she had stopped taking a few days before presentation. There are a few reported cases of pancreatitis associated with phenformin, ${ }^{4}$ but it is not a known complication of metformin therapy. To date only one case of pancreatitis has been reported in association with metformin, but that was secondary to metformin poisoning (overdose). ${ }^{5}$ Available evidence suggests that acute pancreatitis in this patient was probably precipitated by metformin toxicity. The exact mechanism is not known, but toxicity is probably secondary to acinar cell injury leading to intercellular leakage of digestive enzymes from ductules. Cessation of the drug allowed resolution of the pancreatitis.

\section{CONCLUSIONS}

1. Metformin should be prescribed with caution (with regular monitoring of renal function) or, preferably, avoided in patients on other nephrotoxic drugs.

2. Patients with severe lactic acidosis (secondary to metformin) should be promptly referred for haemodialysis. ${ }^{6}$

3. Amylase levels should be measured in this group of patients. Metformin and other drugs should be discontinued in patients with pancreatitis with no identifiable cause.

Correspondence to: Dr Srikumar Mallick, srikumarmallick@hotmail.com

Submitted 29 January 2003

Accepted 1 August 2003

\section{REFERENCES}

1 Lalau JD, Race JM. Lactic acidosis in metformin treated patients. Prognostic value of arterial lactate levels and plasma metformin concentration. Drug Saf 1999;20:377-84.

2 Muchnick JS, Mehta JL. Angiotensin-converting enzyme inhibitor-induced pancreatitis. Clin Cardiol 1999;22:50-1.

3 Borgia MC, Celestini A, Caravella P, et al. Angiotensin-converting-enzyme inhibitor administration must be monitored for serum amylase and lipase in 
order to prevent an acute pancreatitis: a case report. Angiology 2001;52:645-7

4 Graber GM, Marmor BM, Hendel RC, et al. Pancreatitis and severe metabolic abnormalities to phenformin therapy. Arch Surg 1976;111:1014-16.
5 Ben $\mathrm{MH}$, Thabet $\mathrm{H}$, Zaghodoudi I, et al. Metformin associated acute pancreatitis. Vet Hum Toxicol 2002;44:47-8

6 Chang CT, Chen YC, Fang JT, et al. Metformin associated lactic acidosis: case report and literature review. J Nephrol 2002;15:398-402.

\section{IMAGES IN MEDICINE.}

\section{Wound botulism}

A

46 year old man presented with a five day history of nausea and vomiting, blurred vision, slurred speech, and generalised weakness. Two days before admission, he developed diplopia and difficulty breathing. He was an intravenous and subcutaneous, that is, "skin popping" user of black tar heroin, and he was positive for hepatitis C. In the intensive care unit, he was conscious and able to obey commands, although intubated. He had bilateral ptosis and almost complete ophthalmoplegia in all directions (fig 1). Pupils were $4 \mathrm{~mm}$ in diameter, responded sluggishly to light. Bilateral facial weakness was observed. He had mild symmetrical proximal weakness, worse on the right. Reflexes were depressed with flexor plantars. Skin examination showed multiple scars from subcutaneous drug injections with track marks and skin popping lesions (fig 2). Cerebrospinal fluid analysis revealed no white cells and normal protein. Mouse bioassay confirmed Clostridium botulinum type A toxin. He received antitoxin treatment and improved gradually.

Although California has the infamous distinction of reporting the most wound botulism cases in the world, the disease has been increasingly recognised elsewhere due to the widespread use of subcutaneous black tar heroin. ${ }^{1}$ This life threatening infection with severe morbidity should be considered in all patients with history of subcutaneous drug use presenting with generalised weakness. Ophthalmoparesis with sluggish pupils and bulbar dysfunction are important clues to the diagnosis. In addition to supportive measures, searches for abscess and antibiotics are the mainstay of treatment. ${ }^{2}$ Antitoxin treatment has been reported to shorten hospital stays with fewer days on ventilatory support.

R Bhidayasiri Y M Choi

R Nishimura

Department of Neurology, UCLA Medical Center, UCLA School of Medicine, California,

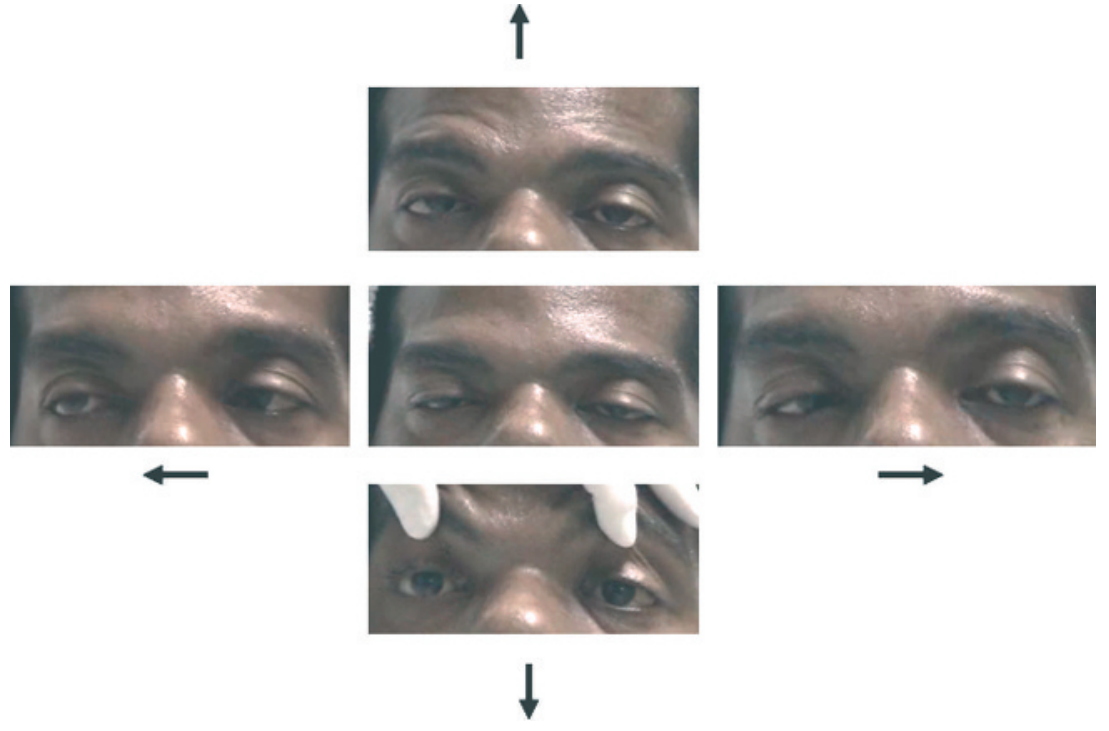

Figure 1 The patient had bilateral proptosis and almost complete ophthalmoplegia in all directions. In addition, bilateral facial weakness was noted (arrows correspond to attempts on direction of gaze).

Correspondence to: $\operatorname{Dr} \mathrm{R}$ Bhidayasiri, Department of Neurology, Reed Neurological Research Institute, UCLA Medical Center, 710 Westwood Plaza, Los Angeles, CA 90095, USA; rbh@ucla.edu

\section{REFERENCES}

1 Passaro DJ, Werner SB, McGee J, et al. Wound botulism associated with black tar heroin among injecting drug users. JAMA 1998;279:859-63.

2 Merrison AFA, Chidley KE, Dunnett J, et al. Wound botulism associated with subcutaneous drug use. BMJ 2002;325:1020-1.

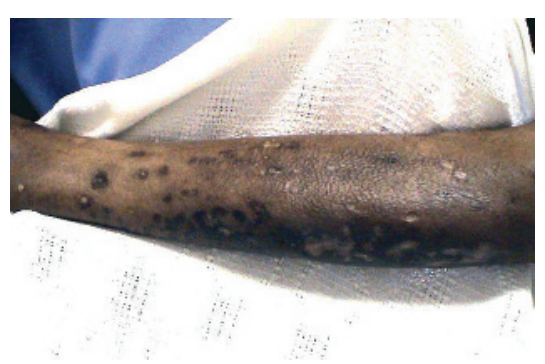

Figure 2 Skin examination of his right leg demonstrated multiple scars from subcutaneous drug injections with track marks and skin popping lesions. 